\title{
Staining and calculus formation after $0.12 \%$ chlorhexidine rinses in plaque-free and plaque covered surfaces: a randomized trial
}

\author{
Fabrício Batistin ZANATTA ${ }^{1,2}$, Raquel Pippi ANTONIAZZI' ${ }^{1}$ Cassiano Kuchenbecker RÖsING ${ }^{2}$ \\ 1- DDS, School of Dentistry, Division of General Dentistry, Franciscan University Center (UNIFRA), Santa Maria, RS, Brazil. \\ 2- PhD, Post-Graduate Program in Dentistry, Lutheran University of Brazil (ULBRA), Canoas, RS, Brazil. \\ Corresponding address: Fabrício B. Zanatta - Rua Tiradentes, 76/801 - Bairro Centro - 97050730 - Santa Maria, RS - Brasil - Phone: +55 5533078026 - \\ Fax: +55 5133384221 - e-mail: fabriciobzanatta@yahoo.com.br
}

Received: February 2, 2009 - Modification: September 5, 2009 - Accepted: September 28, 2009

\section{ABSTRACT}

\begin{abstract}
$\mathrm{O}$ bjectives: Studies concerning side effects of chlorhexidine as related to the presence of plaque are scarce. The purpose of this study was to compare the side effects of $0.12 \%$ chlorhexidine gluconate ( $\mathrm{CHX}$ ) on previously plaque-free (control group) and plaque-covered surfaces (test group). Methods: This study had a single-blind, randomized, split-mouth, 21 days-experimental gingivitis design, including 20 individuals who abandoned all mechanical plaque control methods during 25 days. After 4 days of plaque accumulation, the individuals had 2 randomized quadrants cleaned, remaining 2 quadrants with plaque-covered dental surfaces. On the fourth day, the individuals started with $0.12 \% \mathrm{CHX}$ rinsing lasting for 21 days. Stain index intensity and extent as well as calculus formation were evaluated during the experimental period. Results: Intergroup comparisons showed statistically higher $(p<0.05)$ stain intensity and extent index as well as calculus formation over the study in test surfaces as compared to control surfaces. Thus, $26.19 \%$ of test surfaces presented calculus, whereas calculus was observed in $4.52 \%$ in control surfaces. Conclusions: The presence of plaque increased $0.12 \% \mathrm{CHX}$ side effects. These results strengthen the necessity of biofilm disruption prior to the start of $\mathrm{CHX}$ mouthrinses in order to reduce side effects.
\end{abstract}

Key words: Chlorhexidine. Adverse effects. Staining. Tooth discoloration. Dental calculus.

\section{INTRODUCTION}

Several antimicrobial agents have been incorporated in mouthrinses to improve the outcome of mechanical oral hygiene procedures or even to replace mechanical plaque control. Chlorhexidine gluconate $(\mathrm{CHX})$ is presently the most effective compound having pronounced antimicrobial effects both on Gram-positive and Gram-negative bacteria as well as on fungi and some viruses ${ }^{5,28}$. $\mathrm{CHX}$ is a positively charged bisbiguanide, which can adsorb to different negatively charged sites including mucous membranes, salivary pellicle on teeth and titanium implant surfaces as well as several components of the biofilm on the tooth surfaces such as bacteria, extracellular polysaccharides and glycoproteins ${ }^{15,18,27}$. However, it has not been recommended for long periods because of the observed side effects ${ }^{12}$.

The reported side effects of $\mathrm{CXH}$ are alteration in taste, increase of calculus formation, staining of teeth and mucous membranes and, more rarely, oral mucosa desquamation and parotid swelling ${ }^{11,16,22}$. However, the most obvious and important local side effects are the brown staining of the teeth, restorative materials and dorsum of the tongue ${ }^{2,11}$ as well as supragingival calculus formation ${ }^{6,21,23}$.

Non-enzymatic browning (Maillard reactions) and formation of pigmented metal sulfides are considered the possible mechanisms of tooth discolorations due to $\mathrm{CHX}$ application ${ }^{9,10}$. However, clinical and laboratory studies provided strong evidence that staining is caused by interaction or precipitation of dietary chromogens with locally adsorbed $\mathrm{CXH}^{1,5,27}$. In the presence of food components, $\mathrm{CHX}$ dyes produce colored compounds on hydroxyapatite ${ }^{17}$. Moreover, when associated with tea and coffee intake, brown staining of tooth and acrylic previously exposed to $\mathrm{CXH}$ is more likely to occur ${ }^{3}$. Corroborating these findings, Addy, et al. ${ }^{4}(1979)$ demonstrated that tea, coffee and red wine were particularly chromogenic. 
$\mathrm{CXH}$ has proven in many clinical trials to be effective in reducing the formation of dental plaque and preventing gingivitis ${ }^{13,14,15,22}$. However, calculus formation seems to be increased ${ }^{19,23}$. All previous studies reporting $\mathrm{CHX}$ anti-plaque efficacy and related side effects utilized a previous disruption of present biofilms in all dental surfaces. However, there is a lack of evidence if the presence of biofilm formed on tooth surfaces would increase the side effects of $\mathrm{CHX}$. Zanatta, Antoniazzi and Rösing $^{30}$ (2007) demonstrated that $0.12 \% \mathrm{CHX}$ mouthrinses showed lower anti-plaque and antigingivitis effects over previously plaque-covered surfaces compared to plaque-free surfaces. Thus, the working hypothesis of this trial is that the presence of plaque when starting $\mathrm{CHX}$ rinsing as a sole measurement for plaque control would increase staining and calculus formation. This would indicate the necessity of previous prophylaxis. The presence of plaque could be one predisposing factor to staining, since its components would facilitate staining.

The aim of the present study was to compare the effects of $0.12 \% \mathrm{CXH}$ gluconate on staining and calculus formation in previously plaque-free and plaque covered surfaces, by means of an experimental gingivitis clinical trial.

\section{MATERIAL AND METHODS}

The present study is a randomized split-mouth clinical trial. The study protocol was subjected to and approved by the Ethics Committee of the Lutheran University of Brazil, Canoas, RS (CEP-ULBRA 2006-095H). The ClinicalTrials.gov Identifier's registration is NCT00818376.

\section{Test panel}

The test panel was recruited from the dental students of the Lutheran University of Brazil, Canoas, RS, Brazil. In order to assess the power of the present study, using the power calculation with data from mean calculus formation in the present study at day 25 , taking into consideration the paired design, a mean difference of 0.20 and accepting an a-error of 0.05 , revealed a power of 0.85 .

The mean age of the volunteers was $28.15 \pm 3.15$ years. At recruitment, subjects were asked about their medical and dental histories. Written and oral explanations detailing the study purpose and design were given for each subject. Subjects that preliminarily met inclusion/exclusion criteria were selected for a dental screening appointment. If the subject met all the inclusion/exclusion criteria, an informed consent was handed out and, upon acceptance, signed by the volunteers.

\section{Inclusion criteria}

- Age between 18-35 years;

- Male, in order to avoid hormonal influences during the experimental period, especially concerning gingival inflammation;

- No relevant medical conditions that could interfere on the periodontal health;

- Willingness to comply.

\section{Exclusion criteria}

- Probing pocket depth $>3 \mathrm{~mm}$ and/or clinical attachment loss $>2 \mathrm{~mm}$ at any site;

- Antibiotic and/or anti-inflammatory therapy within 3 months prior to baseline examination;

- Oral mucosal lesions;

- Smokers;

- Need for antibiotic premedication;

- History of hypersensitivity to CHX;

- Any condition device that could act as plaque retentive factor (carious lesions; inadequate restorations; dental implants; orthodontic appliances; fixed or removable prostheses).

\section{Clinical Parameters}

The following clinical parameters were assessed in the order listed below from all teeth, excepting the third higher/lower molar.

- Presence of calculus (C) in all teeth, at 6 sites per tooth (mesiobuccal, mid-buccal, distobuccal, mesiolingual, mid-lingual and distolingual) was scored as a dichotomous index: 0 - Absence of calculus; 1 - Presence of calculus.

- Discoloration Index proposed by Lobene ${ }^{20}$ (1968) and modified by Macpherson, et al. ${ }^{24}$ (2000). This involves visual stain assessment of the buccal/labial and lingual/palatal aspects of the index teeth. The modification consisted of dividing each aspect into 4 separate sites (Figure 1), classified as: - Gingival (G): $2 \mathrm{~mm}$ wide strip running parallel to the gingival margin. The limit towards the incisal edge given by the end of the interdental papilla; - Body (B): Central area of buccal/lingual aspect, between gingival and distal/ mesial sites, extending to incisal edge; - Mesial (M): Visible area between line angle and adjacent tooth, ending at the interdental papilla and starting at gingival site; - Distal (D): as for mesial (M) site.

Stain was recorded using two separate

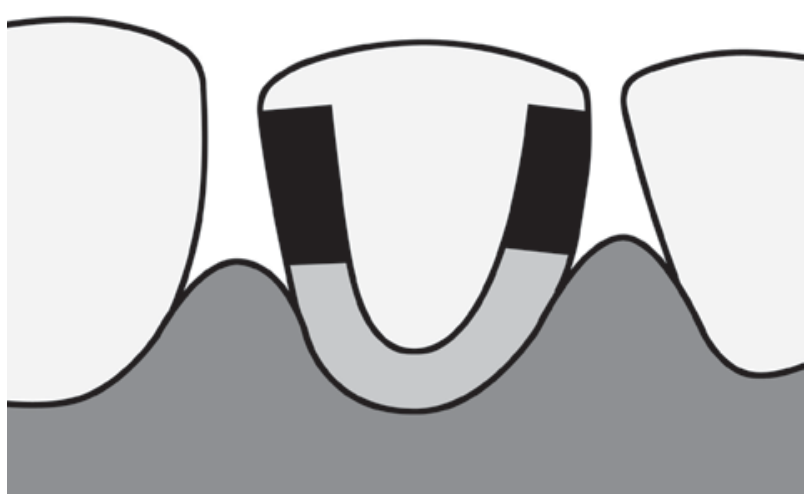

Figure 1- The stain sites of an anterior tooth: Body (White); Gingival (Grey); Mesial and Distal (black) 
characteristics, namely intensity and area (extent). The criteria and codes for intensity were: $0=$ no stain present with the natural tooth coloration; $1=$ slim stain; $2=$ clearly visible stain, orange to brown; $3=$ dark stain, dark brown to black.

The area (extent) of the stain was recorded only if an intensity score of 2 or 3 was given. The area criteria and codes for proximal, gingival and body sites were: $1=$ up to $1 / 3$ of area affected; $2=$ between $1 / 3$ and $2 / 3$ of area affected; $3=$ more than $2 / 3$ of area affected.

\section{Assessmentofintraexaminerreproducibility}

Before starting the trial, multiple sessions of training exercises for clinical parameters were performed. After the examiner was trained, the intra-examiner reproducibility was assessed. For reproducibility, 4 chronic $\mathrm{CHX}$ users, who were not included in the study population, were recruited. After an informed consent, subjects were evaluated two times with one day intermission. In each session, the examiner scored all teeth, except the third molars. The results of presence/absence of calculus and stain index ${ }^{24}$ were analyzed by Kappastatistics. The K-coefficient was 0.96 and 0.68 for calculus and Stain indexes, respectively.

The outline of the experimental procedures is summarized in Figure 2.

To achieve optimal gingival health and to standardize gingival baseline conditions, all subjects participated in a pretrial period during 14 days. Thorough professional scaling and polishing with a rubber cup and prophylactic paste and dental floss was performed. If hygiene was judged unsatisfactory, individual instructions were given on how to improve the performance.

- Baseline: On day zero, the presence of calculus and the stain index were recorded in all subjects. After the recordings, a prophylaxis was performed and detailed explanations to discontinue all the plaque control measurements were provided again.

- Day 4: Each subject had one upper and one lower quadrant randomly assigned by a heads or tails raffle made by the examiner, as "test" (supragingival plaque-covered sites) and the other two quadrants as "controls" (supragingival plaque was professionally removed by the examiner to warrant plaque-free surfaces). After the prophylaxis on control quadrants, the Quigley \& Hein Plaque Index was recorded in all teeth to ensure that control quadrants were plaque-free. Following, all subjects continued the oral hygiene withdrawal for a 21 days and started to rinse twice daily with $15 \mathrm{~mL}$ of a mouthrinse containing $0.12 \%$ CHX (Noplak, Daudt, São Paulo, SP, Brazil).

- Day 11 and 18: Presence/absence of calculus and stain index was recorded in all teeth.

- Day 25: Similar to days 11 and 18. After the recordings, a professional scaling and prophylaxis was performed to remove stain and calculus and all subjects were free to return the normal habits of mechanical plaque control. Gingivitis was treated if necessary.

\section{Blindness}

On days 11,18 and 25 , the examiner was kept unaware of randomization sequence and blinded to which quadrant was test or control. However, because of the nature of the study, it is not possible to assume that the examiner had not become aware of quadrant allocation because plaque accumulation was clinically different between test and control quadrants essentially on days 11 and 18. Whether this problem may have biased the examiner is not ascertained.

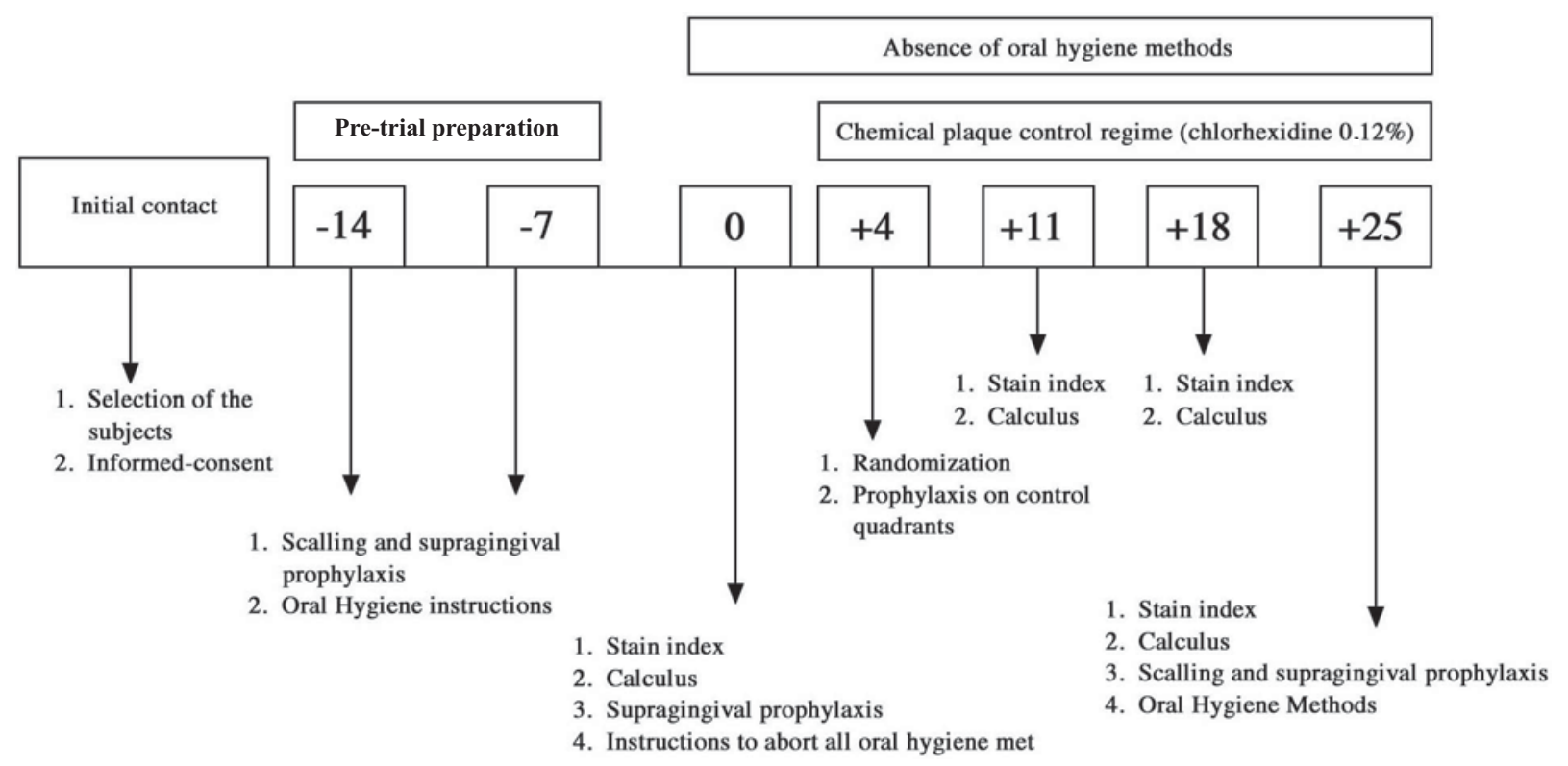

Figure 2- Experimental design 


\section{Compliance}

The necessary volume of $\mathrm{CHX}$ to all the experimental period was $630 \mathrm{~mL}(15 \mathrm{ML}-12 / 12$ h). However, to ensure compliance, more than 630 $\mathrm{mL}$ of $\mathrm{CHX}$ solution was given in flasks with $1 \mathrm{~L}$ volume, with different volumes in each flask. At the end of the experiment, the remaining solution was turned in to check the individual compliance.

\section{Statistical analysis}

Data analysis was performed using commands that take into account clustering of observations within subjects (Stata 9.2 for Windows, Stata Corporation, College Station, TX, USA). A robust variance estimator was used to adjust for the clustering of teeth into individuals. Wald tests were used for comparisons, and the p-value was adjusted for multiple comparisons. The level of significance was set at $5 \%$.

\section{RESULTS}

All 20 subjects who fulfilled all inclusion/ exclusion criteria completed the study. Checking of compliance revealed that all volunteers adhered to the instructions, with the correct amount of left over rinsing solution given back. No other side effects, such as taste alterations, allergic reaction, etc. were observed. Gingival inflammation in this test panel evaluated by the Gingival Index for test was $0.21 \pm 0.02$ and $0.93 \pm 0.03$ at baseline and day 25 , respectively, whereas in control surfaces it was of $0.18 \pm 0.01$ and $0.52 \pm 0.03$ at the same time points. Statistically significant higher values were shown for initially plaque-covered surfaces ${ }^{30}$.

The intensity of stain as assessed by discoloration index did not differ among experimental groups at baseline. When test and control surfaces were compared, higher degrees of staining were present in test surfaces at days 11, 18 and 25 (Figure 3).

The stain area (extent) was recorded only if an intensity score of 2 or 3 was given. Both test and control surfaces didn't show any score 2 or 3 at days 1 and 18. At day 25, the mean extent index was $1.59 \pm 0.13$ on control surfaces and $1.62 \pm 0.09$ on test initially plaque-covered surfaces. These differences weren't statistically significant. The frequency of extent scores on day 25 in test surfaces showed was $8.66 \%$ of the surfaces with score 1 , whereas at control surfaces was of $3.03 \%$. Score 2 was detected in $10.22 \%$ of test surfaces and 3.88 at control surfaces. Differences were also observed concerning the frequency of score $3(0.71 \%$ for test and $0.17 \%$ for control surfaces (Figure 4).

The presence/absence of calculus was analyzed by a dichotomous index. The mean percentage

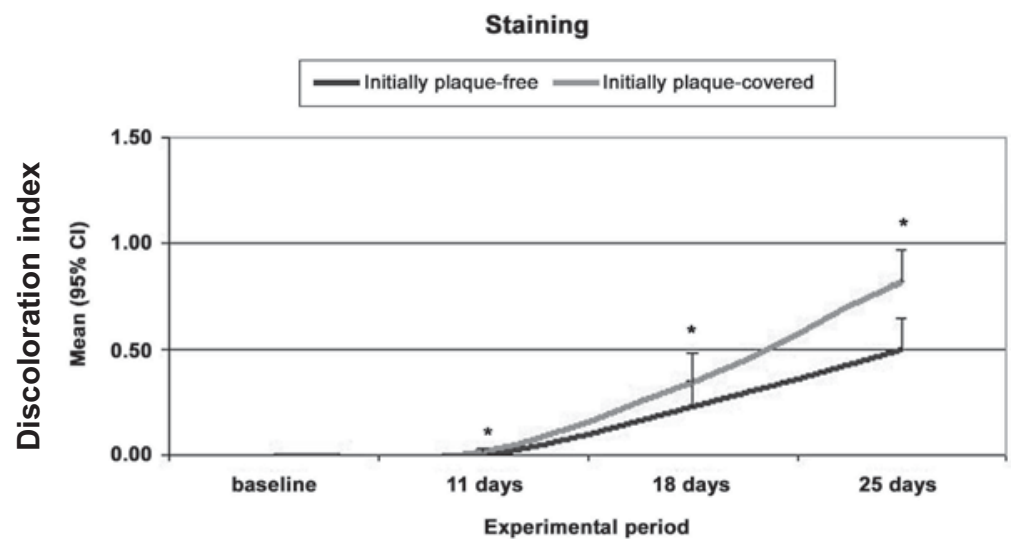

Figure 3- Mean discoloration intensity index in surfaces initially plaque-free or plaque-covered at different experimental periods

* Statistically significant differences between initially plaque-free and plaque-covered surfaces (Wald test, $p=0.05)$

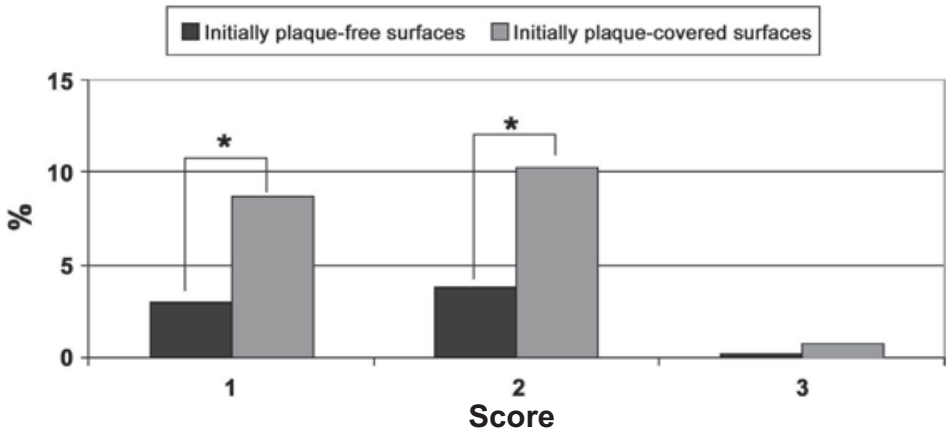

Figure 4- Frequency of sites with scores 1, 2 and 3 of stain extent on day 25

* Statistically significant differences between initially plaque-free and plaque-covered surfaces (Wald test, $p=0.05)$ 


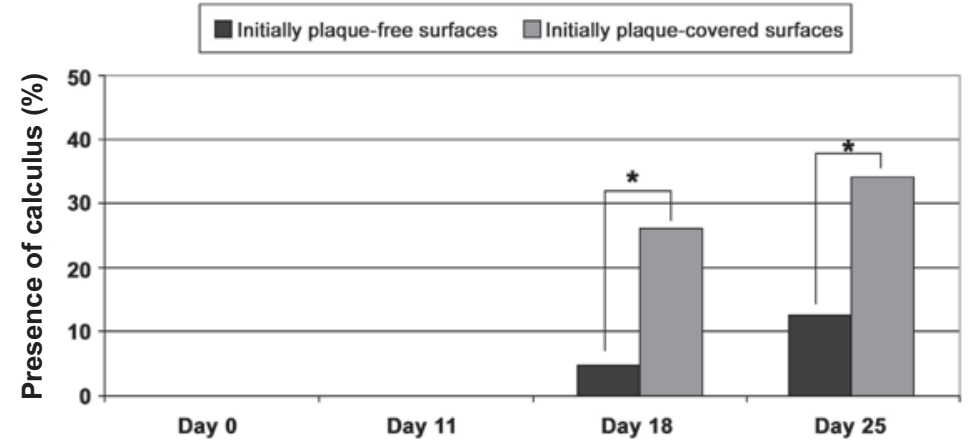

Figure 5- Frequency of sites with presence of calculus

* Statistically significant differences between initially plaque-free and plaque-covered surfaces (Wald test, $p=0.05)$

Table 1- Mean calculus scores on days $0,11,18$ and 25

\begin{tabular}{cllll}
\hline $\begin{array}{c}\text { Presencelabsence of } \\
\text { calculus }\end{array}$ & \multicolumn{2}{c}{ Initially plaque-free surfaces (Control) } & \multicolumn{2}{c}{ Initially plaque-covered surfaces (Test) } \\
& Mean & SE & Mean & SE \\
\hline & & & & 0.00 \\
Day 0 & $0.00 A^{*} a^{* *}$ & 0.00 & $0.00 \mathrm{Aa}$ & 0.00 \\
Day 11 & $0.00 \mathrm{Aa}$ & 0.00 & $0.00 \mathrm{Aa}$ & 0.02 \\
Day 18 & $0.05 \mathrm{Aa}$ & 0.01 & $0.26 \mathrm{Bb}$ & 0.02 \\
\hline Day 25 & $0.12 \mathrm{Ab}$ & 0.02 & $0.34 \mathrm{Bc}$ & \\
\hline
\end{tabular}

*Upper case letters regard to comparison between initially plaque-free surfaces and plaque-covered surfaces in each experimental period. Different letters demonstrate statistically significant differences (Wald test, $p=0.05$ ) ${ }^{* *}$ Lower case letters regard to within group comparison overtime. Different letters demonstrate statistically significant differences (Wald test, $\mathrm{p}=0.05)$. $\mathrm{SE}=\mathrm{Standard}$ Error

of calculus in test and control surfaces is shown in Table 1. Both groups significantly increased calculus from days 11 to 18 and 18 to 25 . When test and control surfaces are compared, statistically significant differences were observed at days18 and 25 (Figure 5).

Comparisons between frequency distribution of sites with calculus did not reveal statistically significant differences among groups at day 11 . However, a higher frequency of sites with calculus was found in the test group as compared to the control group at days 18 (26.19\% vs. $4.52 \%)$ and 25 (34.4\% vs. $12.38 \%$, respectively). These differences were statistically significant (Wald test, $\mathrm{p}<0.05)$.

\section{DISCUSSION}

The purpose of the present study was to investigate if initial professional prophylaxis before the start of $\mathrm{CHX}$ plaque control regime would result in different levels of $\mathrm{CXH}$ side effects (stain and calculus formation). Initially plaque-covered surfaces (test group) showed significantly higher degrees of stain intensity and extent of surface area as well as calculus formation as compared to initially plaque-free surfaces (control group). These results showed that the initial professional prophylaxis seems to have a greater effect in reducing $\mathrm{CXH}$ side effects.

The etiology of extrinsic tooth discolorations due to $\mathrm{CHX}$ rinsing is not fully understood. Nonenzymatic browning reactions (Maillard reactions) and formation of pigmented metal (Fe, Sn) sulfides hypotheses is not supported by direct and conclusive in vivo evidence ${ }^{10}$. However, the main possible mechanism of extrinsic tooth stain is the reaction products of food and beverage, where aldehydes and ketones, natural constituents of various foods, may react with $\mathrm{CHX}$ forming colored products. However, a discoloring capacity of $\mathrm{CHX}$ combined with tea, red wine and coffee has been demonstrated both in vivo ${ }^{2,25}$ and in vitro ${ }^{4}$.

Clinical investigations have demonstrated marked variations in the individual discoloration tendency after a period of $\mathrm{CHX}$ rinsing ${ }^{9,11}$. However, the significant increase in stain in previously plaque-free surfaces has been demonstrated by means of staining indexes ${ }^{9,26}$. Our results are in accordance to these observations. In the present investigation, we found an important effect of the presence of plaque over intensity and extent of tooth staining, since surfaces without supragingival plaque at the beginning of $\mathrm{CHX}$ rinsing showed significant lower staining as compared to surfaces with established plaque over 21 days (Figures 3 
and 4). Thus, initially plaque-covered surfaces were stained earlier than plaque-free surfaces.

There is scarce evidence investigating the presence of plaque over $\mathrm{CHX}$ side effects. Corbet, et al. ${ }^{7}$ (1997) demonstrated in a population with large amounts of plaque and calculus, a significantly higher mean of stain, as assessed by a discoloration index system when compared to a parallel control group (Placebo solution). In our study, we used a randomized split-mouth design. Thus, each subject was its own control. Both test and control surfaces were exposed the same dietary compounds influence. This methodological aspect aimed at reducing inter-individual variability, reducing bias and consequently increasing statistical confidence in the results obtained. Furthermore, a single calibrated clinical examiner (unaware of group allocation) assessed all clinical parameters. The observed results indicate the mechanisms by which staining is higher in plaque-covered surfaces is subject to further investigations. However, a possible explication could be that plaque presence could increase the $\mathrm{CHX}$ salivary protein precipitation.

The increase of calculus formation due to $\mathrm{CHX}$ mouthrinse is a usual finding in early long-term investigations ${ }^{19,23}$. However, short-term studies suggested reduced calculus formation with $\mathrm{CHX}$ rinsing ${ }^{6,21}$. Our data showed that initial prophylaxis was important to reduce calculus formation. Control surfaces showed little amounts of calculus formation over 21 days (about $12 \%$ of surfaces), while test surfaces presented $34.4 \%$ of calculus. Thus, plaque-covered surfaces presented calculus earlier than plaque-free surfaces. These results may be explained by the fact that supragingival calculus is essentially mineralized plaque $^{8}$. The process of mineralization and the $\mathrm{CHX}$ influence on the process are not fully understood, but involves localized supersaturation, nucleation, crystal growth and the transformation of precursor phases such as dicalcium phosphate dehydrate, octacalcium phosphate and amorphous calcium phosphate into more stable, crystalline deposits of hydroxyapatite ${ }^{29}$. The higher tendency to calculus formation in lingual aspects of lower anterior and buccal aspects of upper posterior tooth surfaces may be due to the location of the submandibular and parotid ducts location. In these areas, the abundant supply of urea from the saliva and the high salivary film velocity tend to promote base formation to plaque and calcium phosphate precipitation ${ }^{8}$. Hence, it has been advocated that these locations may be more susceptible to calculus formation because of the low sucrose concentration in saliva with a high saliva film velocity promoting clearance of salivary sugar and acid from plaque, and the higher plaque $\mathrm{pH}$ associated to better access to salivary urea ${ }^{21}$. However, these results have to be interpreted with care because our criteria for calculus included both stained and non- stained calculus. Thus, the increased scores could represent the incremental build-up and hardening of stain in the gingival third of the crowns.

In the present study a modified tooth stain index was used. Macpherson, et al. ${ }^{24}$ (2000) demonstrated that this stain index was more advantageous because it allowed to assess sites with most visible stain separately and offered higher discriminatory power due to a lower coefficient of variation when compared with conventional counterpart ${ }^{20}$. The reason to assess calculus in a dichotomous index was to facilitate the assessment in all present teeth due to the most calculus index use only specific teeth and regions. On the other hand, while these calculus indexes allow for quantification of calculus presence, a dichotomous calculus index shows only calculus presence or absence. However, it did not compromise our results because we aimed at verifying if calculus formation would be present first over plaque-covered surfaces.

$\mathrm{CHX}$ was selected for the antimicrobial treatment because it is the best characterized and most effective chemical antiplaque agent known today ${ }^{13-16,28}$. The concentration selected corresponds to that used clinically for plaque control $(0.12 \%$ or $2 \%)$. Moreover, extensive evidence showed similar plaque reduction and gingival inflammation effectiveness when comparing $0.2 \%$ and $0.12 \%$ $\mathrm{CHX}$ concentrations ${ }^{27}$. Franco Neto, et al. ${ }^{13}$ (2008) recently explored this issue demonstrating that the use of $15 \mathrm{~mL}$ of $0.12 \% \mathrm{CHX}$ rinsing did not differ from $10 \mathrm{~mL}$ of $0.2 \%$ for plaque formation and gingival bleeding in a cross-over double blind 14 days rinsing period study design. It should be emphasized that for some individuals $15 \mathrm{~mL}$ is a high amount and might be uncomfortable. However, evidences show that $0.12 \% \mathrm{CHX}$ seems to reduce stain side effects, mainly tooth staining 27,28 .

The results of the present study are intriguing and should be interpreted from a clinical perspective. Clinicians prescribe rinsing sometimes without professionally cleaning teeth. This could be a practice that would increase the undesirable side effects.

\section{CONCLUSIONS}

The initially plaque-covered and plaquefree surfaces presented tooth stain and calculus formation with different magnitude and timing. The clinical relevance of these results is subject to individual interpretation. However, significant differences in calculus formation and staining were found and the clinical impact consists in an indication that it is always desirable to diminish such side effects. Whenever possible, when starting with $\mathrm{CHX}$ regimes, plaque should be previously removed in the best achievable way in order to reduce side effects. 


\section{ACKNOWLEDGMENTS}

The authors wish to thank the Lutheran University of Brazil, Canoas, RS, and the Franciscan University Center, Santa Maria, RS, for the use of their clinics. The present investigation is independent and was supported by the University and the authors.

Drs. Zanatta, Antoniazzi and Rösing report no conflicts of interest related to this study.

\section{REFERENCES}

1- Addy M, Mahdavi SA, Loyn T. Dietary staining in vitro by mouthrinses as a comparative measure of antiseptic activity and predictor of staining in vivo. J Dent. 1995;23(2):95-9.

2- Addy M, Moran J, Davies RM, Beak A, Lewis A. The effect of single morning and evening rinses of chlorhexidine on the development of tooth staining and plaque accumulation. A blind cross-over trial. J Clin Periodontol. 1982;9(2):134-40.

3- Addy M, Moran J. The formation of stain on acrylic surfaces by the interaction of cationic antiseptic mouthwashes and tea. J Biomed Mater Res. 1984;18(6):631-41.

4- Addy M, Prayitno S, Taylor L, Cadogan S. An in vitro study of the role of dietary factors in the aetiology of tooth staining associated with the use of chlorhexidine. J Periodontal Res. $1979 ; 14(5): 403-10$.

5- Addy M, Wade W, Goodfield S. Staining and antimicrobial properties in vitro of some chlorhexidine formulations. Clin Prev Dent. 1991;13(1):13-7.

6- Cancro LP, Paulovich DB, Klein K, Picozzi A. Effects of a chlorhexidine gluconate mouthrinse on dental plaque and calculus. J Periodontol. 1972;43(11):687-91.

7- Corbet EF, Tam JO, Zee KY, Wong MC, Lo EC, Mombelli AW, et al. Therapeutic effects of supervised chlorhexidine mouthrinses on untreated gingivitis. Oral Dis. 1997;3(1):9-18.

8- Dawes C, MacPherson LM. The distribution of saliva and sucrose around the mouth during the use of chewing gum and the implications for the site specificity of caries and calculus deposition. J Dent Res. 1993;72(5):852-7.

9- Ellingsen JE, Rolla G, Eriksen HM. Extrinsic dental stain caused by chlorhexidine and other denaturing agents. J Clin Periodontol. 1982;9(4):317-22.

10- Eriksen HM, Nordbo $\mathrm{H}$, Kantanen $\mathrm{H}$, Ellingsen JE. Chemical plaque control and extrinsic tooth discoloration. A review of possible mechanisms. J Clin Periodontol. 1985;12(5):345-50.

11- Flötra L. Different modes of chlorhexidine application and related local side effects. J Periodontal Res Suppl. 1973;12(3):414.

12- Flötra L, Gjermo P, Rölla G, Waerhaug J. Side effects of chlorhexidine mouth washes. Scand J Dent Res. 1971;79(2):11925.
13- Franco Neto CA, Parolo CC, Rösing CK, Maltz M. Comparative analysis of the effect of two chlorhexidine mouthrinses on plaque accumulation and gingival bleeding. Braz Oral Res. 2008;22(2):139-44.

14- Gjermo P, Baastad KL, Rölla G. The plaque inhibiting capacity of 11 antibacterial compounds. J Periodontal Res. 1970;5(2):1029.

15- Gjermo P, Bonesvoll P, Rölla G. Relationship between plaqueinhibiting effect and retention of chlorhexidine in the human oral cavity. Arch Oral Biol. 1974;19(11):1031-4.

16- Guimarães AR, Peres MA, Vieira RS, Ferreira RM, RamosJorge $M L$, Apolinário $S$, et al. Self-perception of side effects by adolescents in a chlorhexidine-fluoride-based preventive oral health program. J Appl Oral Sci. 2006;14(4):291-6.

17- Jensen JE. Binding of dyes to chlorhexidine-treated hydroxyapatite. Scand J Dent Res. 1977;85(5):334-40.

18- Kozlovsky A, Artzi Z, Moses O, Kamin-Belsky N, Greenstein RB. Interaction of chlorhexidine with smooth and rough types of titanium surfaces. J Periodontol. 2006;77(7):1194-200.

19- Lang NP, Hotz P, Graf H, Geering AH, Saxer UP, Sturzenberger $O P$, et al. Effects of supervised chlorhexidine mouthrinses in children. A longitudinal clinical trial. J Periodontal Res. 1982;17(1):101-11.

20- Lobene RR. Effect of dentifrices on tooth stains with controlled brushing. J Am Dent Assoc. 1968;77(4):849-55.

21- Löe $H$, Mandell $M$, Derry A, Schott CR. The effect of mouthrinses and topical application of chlorhexidine on calculus formation in man. J Periodontal Res. 1971;6(4):312-4.

22- Löe $H$, Schiött CR. The effect of mouthrinses and topical application of chlorhexidine on the development of dental plaque and gingivitis in man. J Periodontal Res. 1970;5(2):79-83.

23- Löe H, Schiött CR, Glavind L, Karring G, Karring T. Two years oral use of chlorhexidine in man. I. General design and clinical effects. J Periodontal Res. 1976;11(3):135-44.

24- Macpherson LM, Stephen KW, Joiner A, Schafer F, Huntington E. Comparison of a conventional and modified tooth stain index. J Clin Periodontol. 2000;27(11):854-9.

25- Prayitno S, Taylor L, Cadogan S, Addy M. An in vivo study of dietary factors in the aetiology of tooth staining associated with the use of chlorhexidine. J Periodontal Res. 1979;14(5):411-7.

26- Rölla G, Melsen B. On the mechanism of plaque inhibition by chlorhexidine. J Dent Res. 1975;54(Sp Iss):B57-62.

27- Smith RG, Moran J, Addy M, Doherty F, Newcombe RG, Smith RG. Comparative staining in vitro and plaque inhibitory properties in vivo of $0.12 \%$ and $0.2 \%$ chlorhexidine mouthrinses. J Clin Periodontol. 1995;22(8):613-7.

28- Torres SR, Peixoto CB, Caldas DM, Akiti T, Barreiros MGC, Uzeda $M$, et al. A prospective randomized trial to reduce oral Candida spp. colonization in patients with hyposalivation. Braz Oral Res. 2007;21(2):182-7.

29- White DJ, Bowman WD, Nancollas GH. Physical-chemical aspects of dental calculus formation and inhibition: in vivo and in vitro studies. In: Ten Cate JM. Recent advances in the study of dental calculus. Oxford: IRL Press; 1989. p. 175-88.

30- Zanatta FB, Antoniazzi RP, Rösing CK. The effect of $0.12 \%$ chlorhexidine gluconate rinsing on previously plaque-free and plaque-covered surfaces: a randomized controlled clinical trial. J Periodontol 2007;78(11):2127-34. 